\title{
ROLE OF FAT EMBOLI IN DIABETIC RETINOPATHY*
}

\author{
BY \\ C. A. G. $\mathrm{COOK}$ \\ Department of Pathology, Institute of Ophthalmology, University of London
}

THE association between diabetes mellitus and disturbance of fat metabolism has long been recognized, and the lipoidal nature of the diabetic vascular changes has been emphasized by many workers particularly with reference to the retinal and renal changes. It is only in recent years, however, that fat emboli have been reported in association with diabetic vascular disease. Thus Wilens, Elster, and Baker (1951) demonstrated that the deposition of fat in the lesions of diabetic glomerulosclerosis is directly proportional to the severity of the renal involvement. Hartroft (1955) found that fat emboli in choline-deficient rats eventually gave rise to glomerular lesions resembling human diabetic glomerulosclerosis, and Kent (1955) showed that fat emboli indistinguishable from the traumatic variety were to be found much more frequently in diabetics than in non-diabetics.

Retinal vascular lesions following traumatic fat embolism were reported by Czerny (1875), but the possibility that a similar process might play a part in the pathogenesis of diabetic retinopathy has attracted little attention, although it is noteworthy that Morgan (1949) reported that a case of traumatic fat embolism showed a fundus picture not unlike that occasionally seen in the diabetic, and that Urbanek (1933) remarked upon the similarity of the ophthalmoscopic picture seen after fat embolism to that in senile diabetics with arteriosclerosis.

In view of these facts, the present investigation was undertaken to examine microscopically the retinal and renal lesions produced by experimental fat emboli in an attempt to evaluate their possible role in the pathogenesis of diabetic vascular disease.

\section{Material and Methods}

A total of eighteen cats received bi-weekly intravenous injections of fat $(1 \mathrm{ml} . / \mathrm{kg}$. bodyweight) over a period varying from one week to 2 months. The time interval elapsing between the last injection and the immediate post-mortem examination of the retina and kidney also varied from 2 hours to 6 months. Although four varieties of fat were used in different animals (olive oil, arachis oil, and animal fat derived from both the cat and rabbit), no histological differences were noted in either the retinal or renal lesions produced by them. 


\section{Findings}

Retinae examined within 6 hours of a single intravenous injection of fat show that practically the entire retinal arteriolar and capillary bed is filled with a mass of fat disposed in the form of long columns in some vessels and globules of varying size in others. In the latter cases, sharply circumscribed extra-vascular aggregations of fat may be seen in the immediately adjacent retinal stroma (Fig. 1).

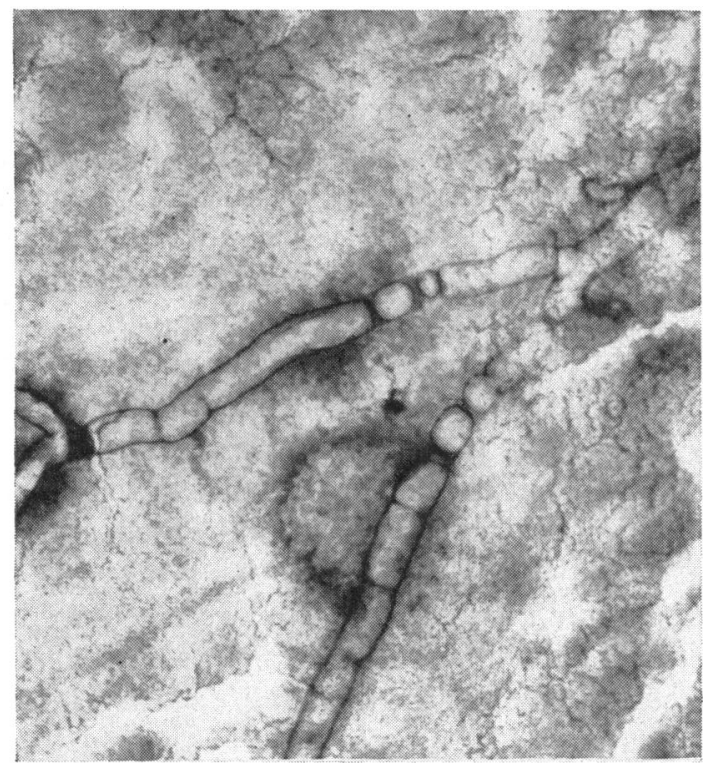

Fig. 1.-Aggregations of fat within the distended arteriolar lumen with localized fatty infiltration into the adjacent retinal tissues. $\times 166$.

In the retinae of animals which had received eight injections of fat, a marked feature was the impaction of isolated fatty globules within the capillary lumen to form multiple localized capillary dilatations, which in many cases were surrounded by a perivascular mantle of fat (Figs 2 and 3, opposite). Although these superficially resembled capillary micro-aneurysms, it must be emphasized that in none of these lesions was any histological evidence of change in the vessel wall found.

Retinae examined after twelve fat injections show that both the intra- and extravascular fatty deposits tend to become progressively more numerous. There were in addition scattered localized areas of retina showing complete capillary collapse, in some of which small partially absorbed haemorrhages were visible. The most noteworthy feature at this stage, however, was the presence of multiple minute fatty globules in both the perivascular spaces of the larger arterioles and also within the arteriolar walls themselves.

A finding of considerable interest was noted in the retinae examined 6 months after the last fat injection. Even after this period scattered intracapillary and extracapillary deposits of fat were still visible, the former in 
Fig. 2.-Retinal capillaries injected with Indian ink. Isolated fat globules are visible both within the capillary lumen and in the perivascular spaces. $\times 93$.
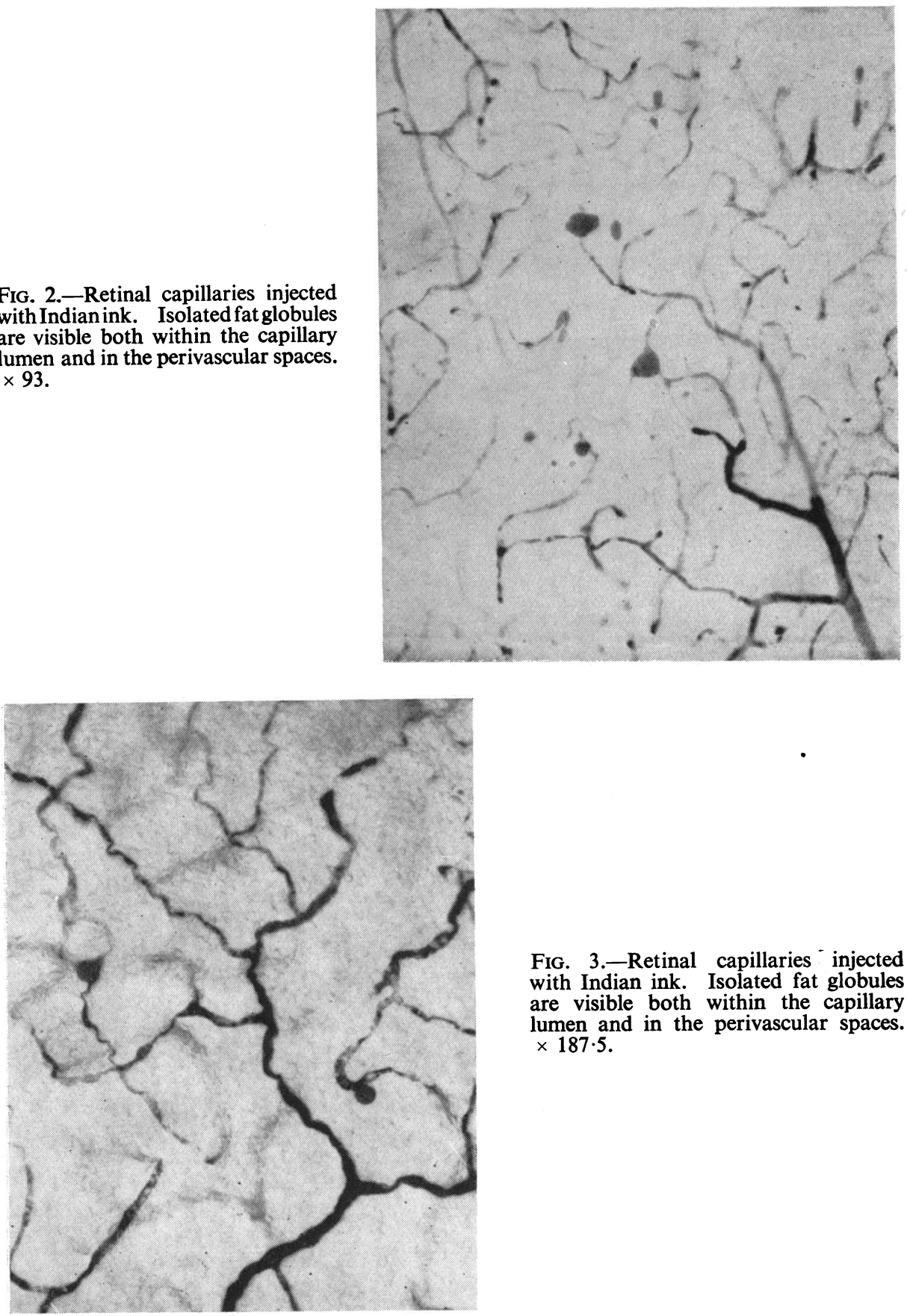

FIG. 3.-Retinal capillaries - injected with Indian ink. Isolated fat globules are visible both within the capillary lumen and in the perivascular spaces. $\times 187 \cdot 5$.

some cases associated with a marked localized capillary dilatation. The deposits along the arterioles however, had completely disappeared and 
although the fatty deposits were in some areas associated with a localized zone of capillary collapse, no evidence of degenerative change in any of the capillary walls was apparent.

The renal embolic lesions produced in this investigation consisted essentially of multiple sharply-circumscribed globules of fat impacted within the glomerular capillaries (Fig. 4). The histological appearances of these glomerular lesions were quite unlike those of diabetic glomerulosclerosis, for not only were the glomerular capillary walls intact but there was also a complete absence of the glomerular nodules pathognomonic of the human renal lesion.

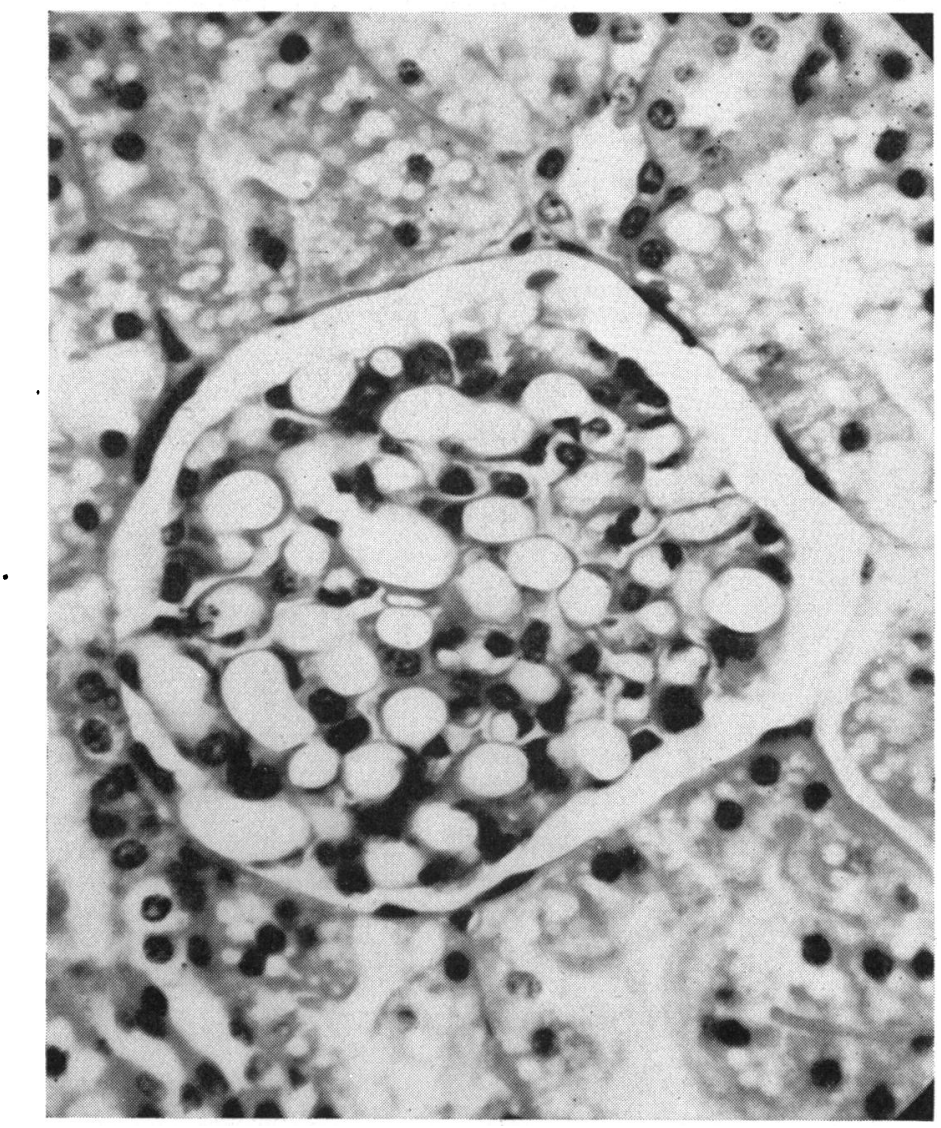

Fig. 4.-Renal glomerulus showing multiple fat globules. $\times 510$.

Discussion

Whilst circumstantial evidence can be adduced directly associating the retinal and renal lesions of diabetes with a disturbance of fat metabolism, the findings of this investigation do not support the hypothesis that such 
lesions can be produced by fat embolism alone. Although the experimentally induced retinal lesions may superficially resemble the diabetic micro-aneurysm, the lack of degenerative and infiltrative changes of the capillary wall must sharply differentiate such inert capillary dilatations from those associated with diabetic vascular disease. These results, however, do not preclude the possibility that the lipoid constituents of the retinal and renal lesions may be directly due to a deposition of plasma fat in a different physico-chemical form than that used in these experiments. Indeed, such a mechanism is possibly suggested by the work of Keiding, Mann, Root, Lawry, and Marble (1952), who found a superficial relationship between the presence of diabetic retinopathy lesions and the elevation in the plasma of macromolecular lipoproteins of the Sf $12-20$ class as identified by the ultracentrifuge.

It must be emphasized, however, that such a postulate as yet entirely lacks either clinical or experimental confirmation, but further investigation into this aspect of the problem might well result in a most valuable addition to. our as yet fragmentary knowledge of the complex factors involved.

\section{REFERENCES}

CzERNY, V. (1875). Berl. klin. Wschr., 12, 593, 605.

Hartroft, W. S. (1955). Amer. J. Path., 31, 381.

Keming, N. R., ManN, G. V., Root, H. F., LaWry, E. Y., and Marble, A. (1952). Diabetes, $1,434$.

Kent, S. P. (1955). Amer. J. Path., 31, 399.

MORGAN, O. G. (1949). Trans. ophthal. Soc. U.K., 69, 441.

UrbaneK, J. (1933). v. Graefes Arch. Ophthal., 131, 147.

Wilens, S. L., Elster, S. K., and BAKeR, J. P. (1951). Ann. intern. Med., 34, 592. 